\title{
Correction to: Reliable and efficient big service selection
}

\author{
Lin Huang ${ }^{1} \cdot$ Qinglin $\mathrm{Zhao}^{2} \cdot{\mathrm{Yan} \mathrm{Li}^{3}}^{3}$ Shangguang Wang ${ }^{1} \cdot \mathrm{Lei} \mathrm{Sun}^{1} \cdot \mathrm{Wu} \mathrm{Chou}^{4}$
}

Published online: 13 November 2017

(C) Springer Science+Business Media, LLC, part of Springer Nature 2017

\section{Correction to: Information Systems Frontiers (2017)}

https://doi.org/10.1007/s10796-017-9767-x

The original version of this article unfortunately contained mistakes in the author spelling. The first author name is misspelled. The correct spelling and author list is presented above.

The online version of the original article can be found at https://doi.org/ 10.1007/s10796-017-9767-x.

Shangguang Wang

sgwang@bupt.edu.cn

Lin Huang

linhuang@bupt.edu.cn

Qinglin Zhao

zqlict@hotmail.com

Yan Li

liyan0417@shisu.edu.cn

Lei Sun

leisun@bupt.edu.cn

Wu Chou

wu.chou@huawei.com

1 State Key Laboratory of Networking and Switching Technology, Beijing University of Posts and Telecommunications, Beijing, China

2 Faculty of Information Technology, Macau University of Science and Technology, Avenida Wei Long, Taipa, Macau, China

3 School of Business and Management, Shanghai International Studies University, Hongkou, Shanghai, China

4 Huawei Technologies Co., Ltd., Shenzhen, China 